\title{
The rubber pencil illusion
}

\author{
JAMES R. POMERANTZ \\ State University of New York, Buffalo, New York
}

\begin{abstract}
When a straight, rigid line segment is put into certain types of motion, it appears to an observer to lose its rigidity and become rubbery, as in the well-known "rubber pencil illusion." The factors controlling this illusion were examined, including the nature of the motion employed (harmonic or linear oscillation), the amplitudes of the translational and rotational components of the motion, and the phase relationship between these two components. The effect is shown to be due to visual persistence. The status of the illusion as a potential counterexample to the rigidity principle (that moving, two-dimensional arrays will be perceived as rigid) is discussed.
\end{abstract}

The "rubber pencil illusion" (RPI) is a striking visual phenomenon that has gone unnoted in the scientific literature on human visual perception, despite its familiarity to many laypeople. Well known as a magician's trick (Gilbert \& Rydell, 1977), it is easily produced by wiggling a pencil that is held loosely and off-center between the thumb and index finger. When executed properly, the pencil seems to become rubbery and to flex conspicuously as it moves. The experiment I report below suggests that the illusion is due to the pattern of visual persistence generated by the dynamic visual image. ${ }^{1}$

The RPI is relevant to the perception of the shapes of moving objects. When we view the image of a moving object projected onto a flat screen, how do we determine if the object is rigid or is changing shape as it moves? Much evidence suggests a bias toward seeing the stimulus as rigid, even if this requires perceiving it to move in the third dimension. For example, if a form presented on a screen changes smoothly from a rectangle to a trapezoid, it will be perceived most often as a rigid rectangle that is rotating in depth, like a door that is swinging open. This effect is, of course, a well-known instance of shape constancy (see Goldstein, 1980, and Hochberg, 1978, for summaries). Similarly, if two dots, placed in diametric opposition, chase one another around an elliptical path, they are perceived as though they were the endpoints of an invisible, rigid rod that is rotating while tilted away from the observer (Johansson, 1974). Finally, if the shadow of a jumbled and rotating ball of wire is projected onto a screen, so that its image is a whirling mass of undulating contours, observers quickly see the display as a rigid structure rotating in depth; this phenom-

This research was supported in part by Research Development and Incentive Funds from the Research Foundation of SUNY. I thank Penny Nuwer for assistance in conducting this experiment. Address requests for reprints to the author at the Department of Psychology, SUNY at Buffalo, 4230 Ridge Lea Road, Buffalo, New York 14226. enon is known as the kinetic depth effect (Wallach \& O'Connell, 1953).

Observations such as these led Johansson (1975) to conclude that "evidently it is obligatory that the spatial relations between ... moving stimuli be perceived as the simplest motion that preserves a rigid connection between the stimuli. The general formula is spatial invariance plus motion." Let us refer to this assertion as the rigidity principle. (A similar notion has been advanced as the "rigidity assumption" by Ullman, 1979.) Besides being an illusion that requires an explanation, the RPI would seem to be a counter-example to the rigidity principle, because the pencil is seen as elastic even though its image is at all times straight.

\section{Purpose of Experiment}

The purpose of this experiment was to explore the RPI in the laboratory, using computer-generated displays shown on the screen of an oscilloscope. With computer-generated displays, the relevant spatial and temporal parameters of the effect can be manipulated systematically and independently to determine which parameters affect the appearance of the illusion.

When the RPI is produced by hand, the pencil oscillates with both a translational (up and down) and a rotational (rocking) component. This occurs because the pencil is held off-center and so its center of gravity is at some distance from where the pencil is grasped; thus, when the hand is moved up and down, a rotational component is introduced automatically into the motion. Both the translational and rotational components are roughly sinusoidal or harmonic. Furthermore, the two components are nearly $180 \mathrm{deg}$ out of phase; that is, when the translational component reaches the top or bottom of its excursion (and thus is at zero velocity), the rotational component reaches its midpoint (and thus is at maximum velocity), and vice versa.

If the pencil is held at its center, it does not ro- 
tate when moved, and no illusion results. If the pencil is held at its tip, it moves both translationally and rotationally, but these two components are then nearly in phase, and little or no illusion results. But when the standard RPI is simulated at the appropriate speed on the oscilloscope, the moving line segment appears to move smoothly and become rubbery, just as in the manually produced RPI.

\section{METHOD}

\section{Subjects}

A group of 16 volunteers recruited from the university community at SUNY at Buffalo served as observers. All had normal or corrected-to-normal vision and all were naive with respect to the purpose of the experiment. Each was tested individually in a single session.

\section{Stimuli and Apparatus}

A set of 48 displays was generated and tested. Each display consisted of a single, straight line placed into motion. The 48

Table 1

Display Parameters and Subjects' Ratings

\begin{tabular}{cccccr}
\hline & & \multicolumn{2}{c}{ Amplitude } & Phase & Mean \\
\cline { 3 - 5 } Number & Type & R & T & $\begin{array}{r}\text { Angle } \\
\text { Rating }\end{array}$ \\
\hline 1 & H & 45 & 0 & & 13.43 \\
2 & H & 90 & 0 & & 16.36 \\
3 & H & 0 & 2.19 & & 2.50 \\
4 & H & 45 & 2.19 & 0 & 13.44 \\
5 & H & 90 & 2.19 & 0 & 32.81 \\
6 & H & 0 & 4.04 & & 4.67 \\
7 & H & 45 & 4.04 & 0 & 12.19 \\
8 & H & 90 & 4.04 & 0 & 20.63 \\
9 & H & 45 & 2.19 & 90 & 28.75 \\
10 & H & 90 & 2.19 & 90 & 50.31 \\
11 & H & 45 & 4.04 & 90 & 20.00 \\
12 & H & 90 & 4.04 & 90 & 44.69 \\
13 & H & 45 & 2.19 & 180 & 32.19 \\
14 & H & 90 & 2.19 & 180 & 50.00 \\
15 & H & 45 & 4.04 & 180 & 37.50 \\
16 & H & 90 & 4.04 & 180 & 68.88 \\
17 & L & 45 & 0 & & 16.88 \\
18 & L & 90 & 0 & & 22.40 \\
19 & L & 0 & 2.19 & & 5.02 \\
20 & L & 45 & 2.19 & 0 & 20.31 \\
21 & L & 90 & 2.19 & 0 & 34.06 \\
22 & L & 0 & 4.04 & & 8.44 \\
23 & L & 45 & 4.04 & 0 & 18.44 \\
24 & L & 90 & 4.04 & 0 & 34.69 \\
25 & L & 45 & 2.19 & 90 & 31.25 \\
26 & L & 90 & 2.19 & 90 & 53.44 \\
27 & L & 45 & 4.04 & 90 & 17.81 \\
28 & L & 90 & 4.04 & 90 & 59.06 \\
29 & L & 45 & 2.19 & 180 & 36.56 \\
30 & L & 90 & 2.19 & 180 & 58.44 \\
31 & L & 45 & 4.04 & 180 & 31.56 \\
32 & L & 90 & 4.04 & 180 & 58.13 \\
\hline
\end{tabular}

Note-Included are descriptions of the motion parameters and the mean rubberiness ratings for the 32 displays tested. The display numbers correspond to the panels in Figure $1 . H$ indicates harmonic motion and $L$, linear. For phase, a blank indicates that phase angle is undefined, since one of the two motion components is absent. Amplitudes and phase angles are given in degrees. $R=$ rotation, and $T=$ translation.

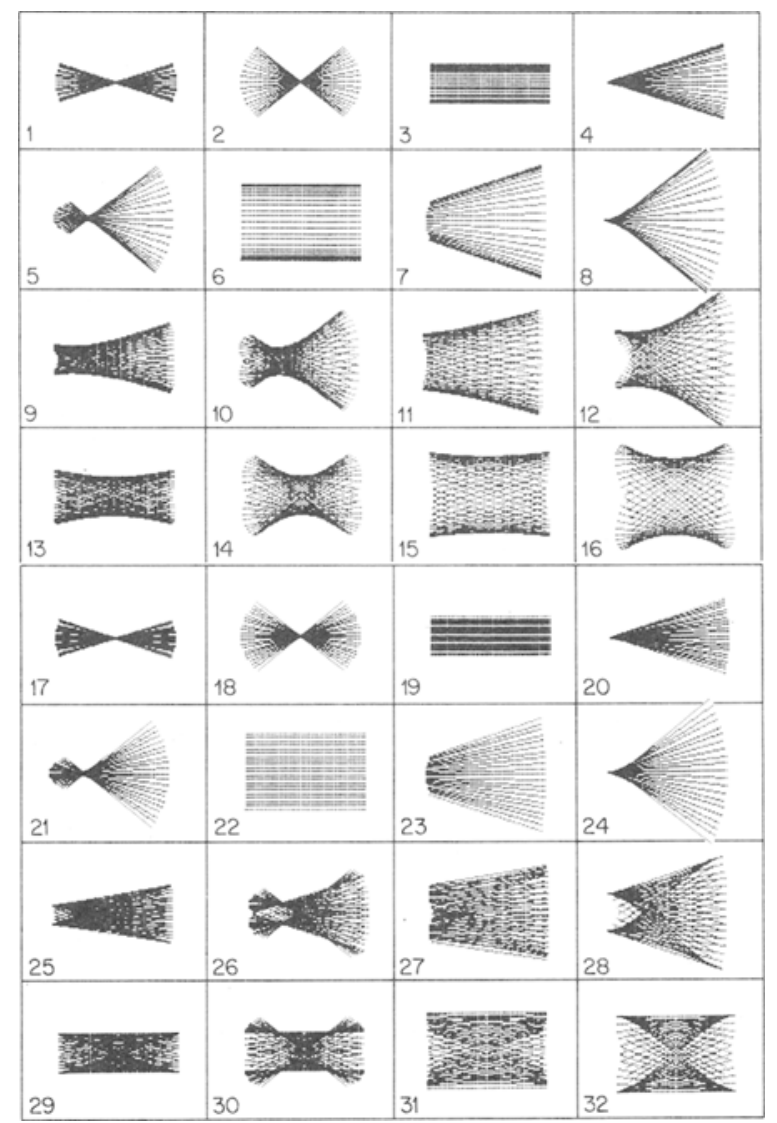

Figure 1. Computer-generated plots of the 32 different displays tested. The panel numbers correspond to the display numbers in Table 1. These reproductions are compressed very slightly in the vertical dimension compared with the actual displays tested. Also, the line segments in these reproductions appear much more jagged than those in the oscilloscopic displays. Each panel shows one complete cycle of motion.

displays resulted from the orthogonal variation of four factors. (1) The motions used were either harmonic (sinusoidal) or linear (triangular wave) oscillations. In the harmonic case, the motion began at zero velocity, increased sinusoidally to a maximum at its midpoint, and then slowed again in a symmetrical fashion to zero velocity before reversing direction. In the linear case, velocity was held constant throughout the trajectory, with instantaneous reversals of direction at the endpoints. (2) The phase angle (synchrony) between the translational and rotational components was set at 0,90 , or $180 \mathrm{deg}$. At 0 deg phase angle, the translational and rotational components were completely in phase and reached their corresponding maxima and minima simultaneously; at $180 \mathrm{deg}$ phase angle, the two components were completely out of phase, as described above. (3) The amplitude of the rotational component was set at 0,45 , or $90 \mathrm{deg}$ of rotation. This parameter refers to the angle through which the stimulus line was rotated (about its midpoint) on the display screen. (4) The amplitude (total vertical excursion) of the translational component was set at $0,2.19$, or $4.04 \mathrm{deg}$ of visual angle.

Of the 54 possible combinations of these four factors, all were used except the six in which the amplitudes of the translational and rotational components would both be set at zero, since this would eliminate all motion from the display. Thus, 48 displays were tested. The frequencies of the translational and rotational components were both held constant at $3.2 \mathrm{~Hz}$. This value was selected to maximize the strength of the illusion: with lower fre- 
quencies (and thus lower velocities) the illusion diminishes, whereas with higher frequencies the moving images become fused and no motion is seen at all.

The moving line was $5.72 \mathrm{deg}$ long and $.41 \mathrm{deg}$ wide. The luminance of the line segments ${ }^{2}$ was $540 \mathrm{~cd} / \mathrm{m}^{2}$ against a background of $17 \mathrm{~cd} / \mathrm{m}^{2}$. Although 48 displays were tested, only 32 of these are different; this was because when the amplitude of either the rotational or translational component is set at zero, phase becomes undefined, and so the triplets of conditions distinguished by phase angle reduce to but one condition. This was the case for 4 of the 8 translational-rotational combinations for both harmonic and linear motion. For design purposes, these redundant conditions were included in the testing, and their repeated rating scores are averaged in Table 1.

Figure 1 shows all 32 different displays as they would appear integrated over a full cycle, as in a long-exposure photograph. Table 1 shows the parameters of each display.

The lines were produced by plotting sets of contiguous points on the oscilloscope. A sheet of frosted acetate placed over the display blurred the lines slightly and made their component points imperceptible. Each line segment was refreshed a fixed number of times before the next line was displayed, and the refreshing was identical for all displays. The oscilloscope (Tektronix Model 5110) was equipped with a fast-decay, P-11 phosphor (decays to $10 \%$ in $.08 \mathrm{msec}$ and to $0.1 \%$ in $20 \mathrm{msec}$ ).

\section{Procedure}

The testing proceeded as follows. The subjects were first seated in a dimly illuminated test chamber and were shown one stimulus (Display 16 in Figure 1) that most closely simulated the traditional, manually produced RPI. All 16 subjects spontaneously reported perceiving the moving line as rubbery. They were then shown all 48 displays, one time each. Four different presentation orders, counterbalanced over subjects, were used. Half the subjects saw a block of 24 linear motion trials first, followed by 24 with harmonic motion; the remaining subjects saw these two blocks in the opposite order. Within blocks, phase and amplitudes of motion were randomly intermixed. The subjects were asked to judge how "rubbery" each display appeared, using a scale where 0 indicated complete rigidity and 100 indicated maximum rubberiness. The subjects viewed the display binocularly from $35 \mathrm{~cm}$.

\section{RESULTS}

The mean numerical response for each display is also shown in Table 1. These means differed significantly by analysis of variance $[F(47,705)=3.73$, $\mathrm{p}<.001]$. The pattern in the data is fairly straightforward. (1) For all 16 subjects, both translational and rotational motion were needed for a strong RPI; when either amplitude was held at zero, little rubberiness was perceived (mean rating of 11.21 when either amplitude was zero vs. 36.03 when neither amplitude was zero). (2) Perceived rubberiness increased with phase angle ( 12 of the 16 subjects showed monotonic increases), with mean ratings of 23.32, 38.16 , and 46.60 at 0,90 , and $180 \mathrm{deg}$ of phase angle, respectively. (3) Rubberiness increased from 5.16 to 21.72 to 37.83 as rotational amplitude increased from 0 to 45 to 90 deg (13 subjects showed a monotonic trend). (4) As translational amplitude increased from 0 to 2.19 to $4.04 \mathrm{deg}$, ratings first increased from 17.27 to 25.78 (13 subjects showed increases) and then dropped insignificantly to 25.69
(9 subjects showed decreases). (5) It mattered little whether the motion was linear or harmonic. Linear motion produced slightly greater rubberiness than harmonic in general (25.50 for linear vs. 21.74 for harmonic, with 11 subjects confirming this difference), but the displays that yielded the very highest ratings had harmonic motion. Moreover, the pattern of results was nearly identical for these two types of motion, in that the eight combinations of rotational and translational amplitudes yielded identical rank orderings with linear and harmonic motion.

\section{DISCUSSION AND CONCLUSIONS}

The meaning of these results is most easily explained by examining Figure 1. Although these figures are static, they reveal important components of subjects' percepts of the actual, moving displays. In Display 16, which produced the highest mean rubberiness rating of 68.44 , motion was harmonic and the translational and rotational components were at maximum amplitude and $180 \mathrm{deg}$ out of phase. The envelope (or scroll) bounding the total trajectory for this display is curved at its top and bottom; it is also denser at its top and bottom than at its center. These densities are inversely related to line velocity and reveal the amount of time the line segment remained in each region of the envelope. A line moving rapidly across the visual field leaves a smeared trace (Burr, 1980) across the retina corresponding to its afterimage or persistence, and the density of this trace is inversely proportional to the velocity of the moving line. A stationary line would yield a maximally dense trace, whereas a line moving at great speed would leave a trace that is barely perceptible. At intermediate speeds, subjects appear to perceive not the true shape of the moving line at any instant (which, of course, is always straight) but rather the shape of the densest regions of the persisting trace inside the motion envelope. In the displays where these regions are curved, rubberiness is perceived, and where they are straight, it is not.

It should be pointed out that even in displays for which no curvature is apparent in Figure 1, some rubberiness was reported by a few subjects. For example, in Displays 1 and 2, which contain only rotational motion (i.e., translational amplitude was zero), mean ratings clearly higher than zero were obtained. With these displays, the moving line does not appear to curve but it does appear to bend sharply at its center, as though it were made from two straight lines hinged together. The nonzero ratings appear to reflect the failure of a few subjects to discriminate between this type of bending and genuine rubberiness.

Note that the RPI does not result exclusively from 
the velocity changes inherent in harmonic motion, in which velocity drops to zero at the turning points. Although these low velocities produce high densities in the retinal smear, recall that linear motion produced a strong RPI too, even though velocity remains constant here. Thus, the particular spatiotemporal properties of the motion trajectory are critical; even with linear motion, the interaction of the translational and rotational components often makes certain points along the line segment come to a momentary standstill.

Finally, notice that the type of curvature apparent in Figure 1 differs for the 32 stimuli. These differences are apparent phenomenally when the moving displays are viewed. For example, the $\mathrm{X}$-shaped configuration in Display 32 yields the impression of a line whose ends bend when they reach the limits of their vertical excursion (described as "clomping feet" by some observers), whereas Display 16, which has an hourglass configuration in Figure 1, is seen to bend at its middle, like a thin metal ruler being flexed.

It seems parsimonious to conclude that the RPI is due to visual persistence, probably occurring early in the visual system, perhaps even in the retina. Temporal integration occurring anywhere in the system could produce the illusion. In fact, it can be produced simply by photographing a waving pencil with a camera using a long exposure duration (although in this case the temporal integration would arise on the film, not in the visual system).

How does this explanation affect the status of the RPI as a counterexample to the rigidity principle? On the one hand, the persistence explanation ascribes the illusion to relatively early, sensory processes, whereas the rigidity principle is presumably intended to explain how ambiguous sensory data are interpreted in later stages of vision. On this ground, one might wish to dismiss the RPI as irrelevant to the rigidity principle; after all, persistence causes the image to bend into a configuration that is inconsistent with rigidity, even for a depthful interpretation. On the other hand, the very existence of the illusion implies that the visual system does not, and perhaps cannot, correct for distortions present in the early visual representation that may affect the perceived shape of a moving object. If rigidity were a more pervasive principle in visual perception, we might expect that mechanisms would have evolved to correct for such sources of error (cf. Burr, 1980). Thus, the rigidity principle can accommodate the RPI, but only at some cost in its generality. In any case, the RPI stands as one of a small number of robust visual illusions that is readily explained by known sensory factors and as one of the most compelling and easily generated demonstrations of visual persistence.

\section{REFERENCES}

Burh, D. Motion smear. Nature, 1980, 284, 164-165.

GILBERT, G., \& RYDELl, W. Great tricks of the master magicians. New York: Golden Press, 1977.

Goldstern, E. B. Sensation and perception. Belmont, Calif: Wadsworth, 1980.

Hochberg, J. E. Perception (2nd ed.). Englewood Cliffs, N.J: Prentice-Hall, 1978.

Johansson, G. Visual perception of rotary motion as transformation of conic sections. Psychologia, 1974, 17, 226-237.

Johansson, G. Visual motion perception. Scientific American, $1975,232,76-88$.

Long, G. M. Iconic memory: A review and critique of the study of short-term visual storage. Psychological Bulletin, 1980, 88, 785-820.

Uluman, S. The interpretation of visual motion. Cambridge, Mass: M.I.T. Press, 1979.

Wallach, H., \& O'Connell, D. N. The kinetic depth effect. Journal of Experimental Psychology, 1953, 45, 205-217.

\section{NOTES}

1. Magicians appear not to have a firm grasp on the visual principles underlying the illusion. For example, Gilbert and Rydell (1977) explain that "there's no trick involved here; it just works that way"' (p. 131).

2. Several subjects were tested over the full range of intensities that the oscilloscope could produce, and the pattern of results was largely unaffected. Although large changes in display intensity will affect the time constant of visual persistence, moderate intensity changes often do not (see Long, 1980, for a review of visual persistence). As a magician's trick, the RPI does not require any special illumination levels.

(Manuscript received October 22, 1982; revision accepted for publication December 15,1982 .) 